Contribution from the Chemistry Departments, University of Coimbra, 3000 Coimbra, Portugal, and The University of Texas at Dallas, Richardson, Texas 75080

\title{
Nuclear Magnetic Resonance and Potentiometric Studies of the Protonation Scheme of a Triaza Triacetic Macrocycle and Its Complexes with Lanthanum and Lutetium
}

\author{
C. F. G. C. GERALDES, ${ }^{* \dagger}$ M. C. ALPOIM, ${ }^{\dagger}$ M. P. M. MARQUES ${ }^{\dagger}$ A. D. SHERRY, ${ }^{* \dagger}$ and M. SINGH ${ }^{\dagger \S}$
}

Received December 28, 1984

\begin{abstract}
The protonation constants of the macrocyclic ligand 1,4,7-triazacyclononane- $N, N^{\prime}, N^{\prime \prime}$-triacetic acid (NOTA) have been measured by potentiometry, and the protonation sequence of the various amino and carboxylate groups of NOTA has been studied in $\mathrm{D}_{2} \mathrm{O}$ as a function of $\mathrm{pD}$ from the chemical shifts of the nonlabile protons. Shielding constants for protonation of the amino groups were determined in a NMR study of the triaza macrocyclic amine, its trimethylated analogue, and NOTA and compared with values reported for linear polyamino polycarboxylates and cyclic tetraaza tetracarboxylate ligands. The results indicate that two nitrogens of NOTA are protonated at higher $\mathrm{pH}$ than the carboxylate groups. The last nitrogen is protonated only at very low $\mathrm{pH}$. The sequence of protonation of NOTA supports the formation of hydrogen bonds between two protonated nitrogens and the adjacent two nonprotonated carboxylates. The ${ }^{1} \mathrm{H}$ and ${ }^{13} \mathrm{C}$ spectra of the $\mathrm{La}$ (NOTA) and Lu(NOTA) species were studied as a function of $\mathrm{pH}$ and temperature. The aqueous complexes show spectra characteristic of flexible triaza macrocycles, displaying fast interconversion between the two staggered $\delta$ and $\lambda$ conformations of the ethylenediamine rings even at room temperature. Above $\mathrm{pH} 9.5$, the La(NOTA) and Lu(NOTA) hydroxo complexes start to form and their spectra show evidence of greater rigidity as at room temperature the $\delta / \lambda$ ethylenediamine ring conformational interconversions in the Lu(NOTA) hydroxo complex are slow on the NMR time scale.
\end{abstract}

Current interest in the complexation properties of macrocycles showing remarkable cation-binding selectivity ${ }^{1}$ has led to the synthesis and study of a large number of new macrocycles derived from cyclic polyaza and cyclic polyaza polyoxa ligands with ionizable functions such as $\beta$-diketonate, ${ }^{2}$ diphenols, ${ }^{3}$ or carboxylate groups. ${ }^{4-7}$ Some of these macrocycles should be very useful as models of certain biological systems and as possible reagents for complexometric titrations. Increased selectivity to cation complexation should also be achieved by these ligands because of the combined effects of internal cavity size, rigidity, and nature of donor atoms and of $\mathrm{pH}$-selective coordinating groups.

The purpose of the present work is twofold. First, the protonation constants and the protonation scheme of the macrocyclic triaza ligand 1,4,7-triazacyclononane- $N, N^{\prime}, N^{\prime \prime}$-triacetic acid (1, NOTA) were obtained with use of potentiometric titrations and ${ }^{1} \mathrm{H}$ NMR $\mathrm{pH}$ titrations. To help interpret the NOTA NMR results, we also studied the cyclic triamine 2 and the $\mathrm{N}$-methylated triaza macrocycle 3. The protonation shifts measured for these cyclic amines were used in a quantitative study of the proton distribution at the various basic sites of NOTA. The conclusions obtained for NOTA were compared with results for noncyclic polyamino polyacetic acids ${ }^{8-10}$ and for the cyclic tetraaza ligands $1,4,7,10$-tetraazacyclododecane- $N, N^{\prime}, N^{\prime \prime}, N^{\prime \prime \prime}$-tetraacetic acid (DOTA) and 1,4,8,11-tetraazacyclotetradecane- $N, N^{\prime}, N^{\prime \prime}, N^{\prime \prime \prime}$ tetraacetic acid (TETA). ${ }^{6,7,11}$ The quantitative determination of the protonation sequence of polyamino polycarboxylic acids such as these should provide important information for the study of their complex equilibria with metal ions as a function of $\mathrm{pH}$.

Second, the structural and dynamic properties of the NOTA complexes with $\mathrm{La}^{3+}$ and $\mathrm{Lu}^{3+}$ were investigated as a function of $\mathrm{pH}$ and temperature in order to assess the potential usefulness of paramagnetic $\operatorname{Ln}($ NOTA) complexes as NMR structural and conformational probes in solution. ${ }^{12}$ The results thus obtained were also compared with similar studies on the analogous DOTA complexes. . $^{13,14}$

\section{Experimental Section}

1. Synthesis and Characterization of the Ligands. Synthesis of the trihydrobromide of the cyclic triamine 2 (1,4,7-triazacyclononane, [9]ane $\mathrm{N}_{3}$ ) was carried out as described in the literature. ${ }^{15-18}$ Macrocycle $3\left(\mathrm{Me}_{3}[9] \mathrm{aneN}_{3}\right)$ was obtained by treating the trihydrobromide of $\mathbf{2}$ with $n$-butyllithium (6 equiv) in dry ether. Quenching with excess iodomethane gave the expected compounds in nearly quantitative yields (yields of $90 \%$ ).

* To whom correspondence may be addressed.

${ }^{+}$University of Coimbra.

\# The University of Texas at Dallas.

\& Permanent address: Guru Nanak Dev University, Amritsar, India.
The ligand NOTA was prepared from the trihydrobromide of the cyclic triamine 2 by adding bromoacetic acid with use of published procedures. ${ }^{13}$ After the reaction was complete, the $\mathrm{pH}$ of the reaction mixture was adjusted to 3.5 with concentrated $\mathrm{HBr}$. White crystals, obtained after adding ethanol and cooling, were washed with ethanol and dried under vacuum at $70^{\circ} \mathrm{C}$ (yield $90 \%$ ). Chemical analysis of the crystals gave the molecular formula $\mathrm{C}_{12} \mathrm{H}_{21} \mathrm{O}_{6} \mathrm{~N}_{3} \cdot 2 \mathrm{NaBr} \cdot 3 \mathrm{H}_{2} \mathrm{O}$. Anal. Calcd for $\mathrm{C}_{12} \mathrm{H}_{21} \mathrm{O}_{6} \mathrm{~N}_{3} \cdot 2 \mathrm{NaBr} \cdot 3 \mathrm{H}_{2} \mathrm{O}: \mathrm{C}, 25.58 ; \mathrm{H}, 4.79 ; \mathrm{N}, 7.46 ; \mathrm{Br}$, $28.42 ; \mathrm{Na}, 8.17$. Found: C, 25.39; H, 4.89; N, 7.43; Br, 28.44; Na, 8.00.

2. Potentiometric Titrations. Potentiometric measurements and calculation of the protonation constants of NOTA were carried out in the laboratory of Dr. G. R. Choppin, Florida State University, Tallahassee, FL. Measurements were carried out in solutions held at a constant ionic strength of $0.1 \mathrm{M}$ with $\mathrm{NaClO}_{4}$.

3. NMR Spectral Measurements. Solutions of ligands $(0.1 \mathrm{M})$ for NMR pH titrations were made up in $\mathrm{D}_{2} \mathrm{O}(99.7 \%$ from Wilmad Glass $\mathrm{Co}$.), and the $\mathrm{pD}$ was adjusted with $\mathrm{DCl}$ or $\mathrm{CO}_{2}$-free $\mathrm{NaOD}$ (Sigma). The final $\mathrm{pH}$ was determined with a Radiometer PHM $64 \mathrm{pH}$ meter equipped with a Philips GA 110-NS electrode and with use of the equation $\mathrm{pD}=\mathrm{pH}+0.4 .^{19}$ The hydrogen electrode used in the present

(1) Kolthoff, I. M. Anal. Chem. 1979, 15, 1R. Izatt, R. M., Christensen, J. J., Eds. "Synthetic Multidentate Macrocyclic Compounds"; Academic Press: New York, 1978

(2) Alberts, A. H.; Cram, D. J. J. Am. Chem. Soc. 1979, 101, 3545.

(3) Weitl, F. L.; Raymond, K. N.; Smith, W. L.: Howard. T. R. J. Am. Chem. Soc. 1978, 100, 1170 .

(4) Takagi, M.; Tazaki, M.; Ueno, K. Chem. Lett. 1978, 1179.

(5) Häflinger, H.; Kaden, A. Helv. Chim. Acta 1979, 62, 683. Stetter, H. S.; Frank, W. Angew. Chem., Int. Ed. Engl. 1976, 15, 686.

(6) Desreux, J. F.; Merciny, E.; Loncin, M. F. Inorg. Chem. 1981, 20, 987.

(7) Delgado, R.; Frausto da Silva, J. J. R. Talanta 1982, 29, 815.

(8) Sudmeier, J. L.; Reilley, C. N. Anal. Chem. 1964, 36, 1699, 1707.

(9) Tackett, J. E.; Sawyer, D. T. Inorg. Chem. 1964, 3, 304. Kula, R. J.; Sawyer, D. T. Ibid. 1964, 3, 458.

(10) Letkeman, P.; Martell, A. E. Inorg. Chem. 1979, 18, 1284. Letkeman, P.; Westmore, J. B. Can. J. Chem. 1971, 49, 2086.

(11) Ascenso, J. R.; Delgado, R.; Frausto da Silva, J. J. R., submitted for publication in J. Chem. Soc., Dalton Trans.

(12) Sherry, A. D.; Singh, M.; Geraldes, C. F. G. C., submitted for publication in J. Magn. Reson.

(13) Desreux, J. F. Inorg. Chem. 1980, 19, 1319.

(14) Bryden, C. C.: Reilley, C. N.; Desreux, J. F. Anal. Chem. 1981, 53. 1918.

(15) Richman, J. E.; Atkins, T. J. J. Am. Chem. Soc. 1974, 96, 2268.

(16) Atkins, T. J.; Richman, J. E.; Oetle, W. F. Org. Synth. 1978, 58, 86.

(17) White, D. W.; Karcher, B. A.; Jacobson, P. A.; Verkade, J. G. J. Am. Chem. Soc. 1979.101, 4921 .

(18) Martin, A. E.; Ford, T. M.; Bulkowski, J. E. J. Org. Chem. 1982, 47. 412 .

(19) Mikkelsen, K.: Nielsen, S. O. J. Phys. Chem. 1960, 64, 632. 
Table I. Protonation Constants $\left(25^{\circ} \mathrm{C}\right)$ of Macrocyclic Complexons and of the Corresponding Macrocyclic Amines

\begin{tabular}{cccccrr}
\hline & {$[9]$ aneN $_{3}{ }^{a}$} & NOTA $^{b}$ & {$[12]$ aneN $_{4}{ }^{c}$} & DOTA $^{d}$ & {$[14]$ aneN $_{4}{ }^{e}$} & TETA $^{d}$ \\
\hline $\log K_{1}$ & 10.42 & $11.3 \pm 0.1$ & 10.7 & 11.08 & 11.58 & 11.56 \\
$\log K_{2}$ & 6.82 & $5.59 \pm 0.02$ & 9.7 & 9.23 & 10.62 & 10.18 \\
$\log K_{3}$ & very small & $2.88 \pm 0.02$ & 1.73 & 4.24 & 1.61 & 4.05 \\
$\log K_{5}$ & & $f$ & 0.94 & 4.18 & 2.41 & 3.38 \\
$\log K_{5}$ & & $f$ & & 1.88 & 2.17 \\
$\log K_{6}$ & & $f$ & & 1.71 & 1.42
\end{tabular}

${ }^{a}$ Taken from ref $20\left(0.1 \mathrm{M} \mathrm{KNO}_{3}\right)$. ${ }^{b}$ Present work $(0.1 \mathrm{M} \mathrm{NaClO})$. The errors represent the mean deviations in the protonation constants obtained from multiple titrations. $\log K_{1}$ was determined from NMR titrations. ' Taken from ref $21 .{ }^{d}$ Taken from ref $6(1 \mathrm{M} \mathrm{NaCl})$. ${ }^{e}$ Taken from ref $22\left(0.5 \mathrm{M} \mathrm{KNO}_{3}\right)$. fNot measured.



1

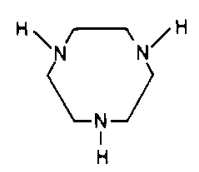

2

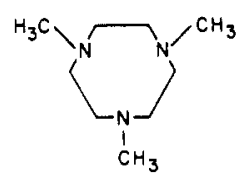

3

Figure 1. Structures of the macrocyclic ligands in this study.

work allows a reliable and accurate determination of the proton activity over an extended $\mathrm{pH}$ range. For some of the $\mathrm{pH}$ titrations, NOTA was purified of existing cations with use of a Dowex 1X8-200 cation-exchange resin (from Sigma) in the $\mathrm{H}^{+}$form and eluted with $1 \mathrm{M} \mathrm{HCl}$. It was then titrated to the basic form in a NMR tube with use of $\mathrm{CO}_{2}$-free potassium or tetramethylammonium hydroxide solutions (from BDH and Sigma, respectively). La(NOTA) and Lu(NOTA) solutions were prepared by mixing appropriate aliquots from stock solutions of lanthanide chlorides and NOTA. Unlike DOTA,${ }^{13}$ the $1: 1 \operatorname{Ln}$ (NOTA) complexes show fast kinetics of complexation.

${ }^{1} \mathrm{H}$ and broad-band proton-decoupled ${ }^{13} \mathrm{C}$ NMR spectra were obtained at 200 and $50.1 \mathrm{MHz}$, respectively, on either a JEOL FX-200 or Varian $\mathrm{XL}-200$ spectrometer. Proton shifts were referenced to $\mathrm{Me}_{4} \mathrm{Si}$ with use of a drop of tert-butyl alcohol as an internal standard. Resonance peaks were measured with a precision better than $0.01 \mathrm{ppm}$. Probe temperatures were accurate to $\pm 1{ }^{\circ} \mathrm{C}$.

\section{Results and Discussion}

1. Ligand Protonation Constants. Table I shows the values of the protonation constants of NOTA, calculated from potentiometric titration curves obtained in the $\mathrm{pH}$ interval 2.0-10.0. This table also compares the protonation constants of NOTA with the literature values for the corresponding macrocyclic triamine, [9] ane $\mathrm{N}_{3}$, and includes a similar comparison of literature values for the macrocyclic tetraaza ligands DOTA and TETA with [12] ane $\mathrm{N}_{4}$ and [14] aneN $\mathrm{N}_{4}$. Although the first protonation constant for NOTA could not be determined in this work by potentiometry, $\log K_{1}=11.3 \pm 0.1$ is obtained from the NMR pH titration of NOTA. As in the case of DOTA and TETA, ${ }^{6}$ we observe a good correlation between the two highest protonation constants of NOTA and its corresponding cyclic triamine, but the remaining $\log K$ 's differ considerably. This data suggests that NOTA, like DOTA and TETA,${ }^{6}$ feature nitrogen atoms that are less basic than carboxylate groups, as opposed to linear amines like EDTA, where all nitrogen atoms are protonated before the carboxylate groups. ${ }^{8-10}$ Notice also that $\log K_{3}=2.88$ for NOTA is similar to $\log K_{3}=$ 2.60 for EDTA while $\log K_{3}$ values for DOTA and TETA are nearer to the $\mathrm{p} K_{\mathrm{a}}$ of acetic acid $\left(\log K_{1}=4.8\right){ }^{6}$

2. NMR pH Titrations. The macroscopic protonation constants in Table I by themselves do not indicate the sequence of ligand

(20) Yang, R.; Zompa, L. J. Inorg. Chem. 1976, 15, 1499.

(21) Kodama, M.; Kimura, E. Yuki Gosei Kagaku Kyokaishi 1977, 35, 632. protonation sites. This microscopic protonation scheme can, however, be obtained by following the NMR chemical shifts of the ligand methylenic protons as a function of $\mathrm{pH}$. This is based on previous observations that the protonation of a basic site of a polyamino polycarboxylate compound in aqueous solution leads to a deshielding of the adjacent methylene protons. ${ }^{8}$ If it is assumed that contributions from the protonation of $N$ basic sites close to a methylene group are additive, then the chemical shift $\Delta \delta_{i}$ of the NMR peak of that methylene group can be approximated as ${ }^{8}$

$$
\Delta \delta_{i}=\sum_{j=1}^{N} C_{i j} f_{j}
$$

where $f_{j}$ is the protonation fraction of the $j$ th basic site, often expressed as a percentage of protonation, and $C_{i j}$ is the protonation shift or shielding constant of the $i$ th resonance for protonation of the $j$ th basic site. Also, if $n$ equiv of acid are added per mole of a ligand with $N$ basic sites, we have

$$
n=\sum_{j=1}^{N} \alpha_{j} f_{j}
$$

where $\alpha_{j}$ is the number of equivalent sites of type $j$. The combination of eq 2 with $m$ equations of the (1) type obtained for the $m$ NMR ligand peaks leads to a set of simultaneous linear equations that can be solved ${ }^{8}$ to obtain the percent protonation $f_{j}$ by standard least-squares techniques, provided the shielding constants are known.

The additivity rule implied by eq 1 only holds in a polyfunctional molecule like a polyamine polycarboxylate if the various substituents maintain constant average relative orientations throughout the whole pH range. ${ }^{8}$ However, linear and especially cyclic molecules of this kind do not obey that condition, ${ }^{6,8,11}$ causing long-range shielding effects of magnetically anisotropic groups, such as hydrogen-bonded carboxylates, ${ }^{6,8}$ or long-range electric field effects of protonated amine groups locked in low-energy conformations which change the $C_{i j}$ constants, e.g. with $\mathrm{pH}$. Consequently, deviations from the protonation shift additivity have been observed. ${ }^{6,8}$ An empirical procedure ${ }^{6,8}$ to interpret the observed protonation shifts of both linear and cyclic polyamines and complexons has been to use different $C_{i j}$ constants for each set of related compounds, derived from analysis of the protonation shifts of simpler related systems taken as models. Table II summarizes a set of relevant published $C_{i j}$ constants obtained for both linear ${ }^{8}$ and cyclic ${ }^{6,11}$ compounds containing carboxylic and amino groups. In our study of the protonation sites of NOTA, we started by studying the protonation scheme of the triaza macrocycles 2 and 3 , considered as good models for the analysis of the more complex NOTA system, in order to determine if yet new $C_{i j}$ parameters should be used.

Triaza Macrocycles 2 and 3. A plot of the chemical shift values of the single NMR ethylenic resonance of [9]ane $\mathrm{N}_{3}(2)$ as a function of $\mathrm{pH}$ shows three inflections centered at $\mathrm{pH} 10.4,6.9$, and $\simeq 0.4 \pm 0.2$ (data not shown). These values correlate quite well with the published protonation constants of [9] ane $\mathrm{N}_{3}$ (Table I) and reflect therefore a stepwise protonation of $\mathrm{L}$ with formation of the species $\mathrm{HL}^{+}, \mathrm{H}_{2} \mathrm{~L}^{2+}$, and $\mathrm{H}_{3} \mathrm{~L}^{3+}$. In each protonation step there is equal simultaneous partial protonation of each ring nitrogen. The fully protonated form of compound 2 exists only in very acidic media, due to strong repulsions of the three protonated 
Table II. Methylenic and Methyl Proton Shielding Constants (ppm) Calculated for Various Cyclic Polyamines ${ }^{f}$

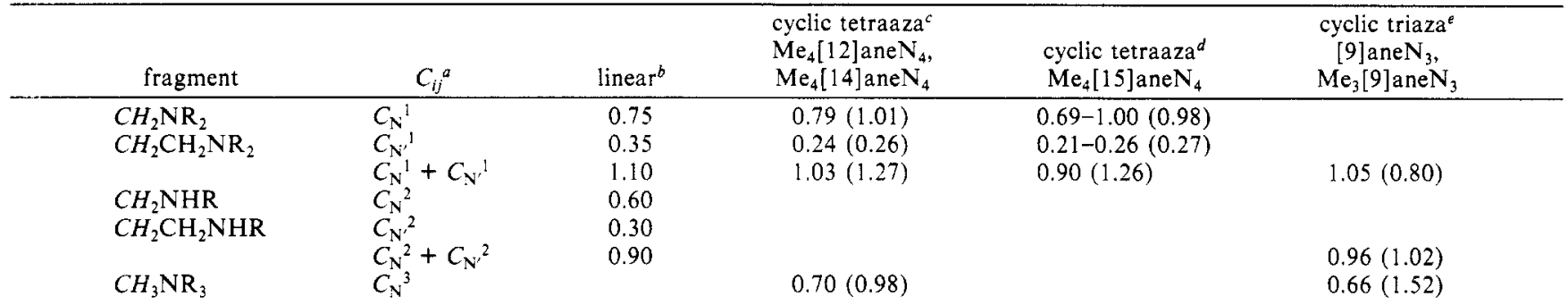

${ }^{a}$ The subscripts $\mathbf{N}$ and $\mathbf{N}^{\prime}$ refer to the shielding of the $\alpha$ and $\beta$ protons, respectively, while the superscripts 1 and 2 refer to the type of fragment involved (tertiary vs. secondary, respectively) as indicated to the left. $C_{\mathrm{N}}{ }^{3}$ refers specifically to the shielding of the methyl protons in the tertiary cyclic methylamine structures. ${ }^{b}$ Taken from ref 8 . ' Taken from ref $6 .{ }^{d}$ Taken from ref 11 . ${ }^{e}$ This work. ${ }^{f}$ Low-pH values are in parentheses.

Table III. Percent Protonation of the Amino Groups $\left(f_{\mathrm{N}}\right)$ and the Carboxylate Groups $\left(f_{\mathrm{O}}\right)$ in the Macrocycles NOTA and DOTA $\left(C_{\mathrm{O}}=0.20\right.$ ppm)

\begin{tabular}{|c|c|c|c|c|c|c|c|c|c|c|}
\hline \multirow[b]{3}{*}{$n$} & \multicolumn{8}{|c|}{ NOTA } & & \\
\hline & \multicolumn{2}{|c|}{$a$} & \multicolumn{2}{|c|}{$b$} & \multicolumn{2}{|c|}{$c$} & \multicolumn{2}{|c|}{$\bar{d}$} & \multicolumn{2}{|c|}{ DOTA $^{e}$} \\
\hline & $f_{\mathrm{N}}$ & $f_{0}$ & $\overline{f_{\mathrm{N}}}$ & $f_{0}$ & $f_{N}$ & $f_{0}$ & $f_{\mathrm{N}}$ & $f_{0}$ & $f_{\mathrm{N}}$ & $f_{0}$ \\
\hline 1 & $43 \pm 4$ & $-20 \pm 20$ & $37 \pm 5$ & $-17 \pm 20$ & $41 \pm 6$ & $-19 \pm 20$ & $33 \pm 1$ & $2 \pm 2$ & $28 \pm 16$ & $-6 \pm 12$ \\
\hline 2 & $79 \pm 10$ & $-36 \pm 40$ & $68 \pm 10$ & $-23 \pm 40$ & $96 \neq 30$ & $-33 \pm 25$ & $61 \pm 2$ & $0 \pm 6$ & $51 \pm 10$ & $3 \pm 5$ \\
\hline 3 & $63 \pm 4$ & $28 \pm 15$ & $68 \pm 4$ & $23 \pm 15$ & $113 \pm 10$ & $-1 \pm 25$ & $67 \pm 2$ & $36 \pm 7$ & & \\
\hline 4 & & & & & & & & & $52 \pm 2$ & $48 \pm 2$ \\
\hline 5 & $81 \pm 8$ & $63 \pm 35$ & $89 \pm 5$ & $63 \pm 30$ & $147 \pm 15$ & $-31 \pm 60$ & $87 \pm 1$ & $80 \pm 1$ & & \\
\hline 6 & & & & & & & & & $58 \pm 7$ & $92 \pm 6$ \\
\hline
\end{tabular}

${ }^{a}$ Used $C_{\mathrm{N}}{ }^{1}=0.79(1.01)$ and $C_{\mathrm{N}^{\prime}}{ }^{1}=0.24(0.26)$ from ref $6 .{ }^{b}$ Used $C_{\mathrm{N}}{ }^{1}=0.91$ and $C_{\mathrm{N}^{\prime}}{ }^{1}=0.27$ from ref 11 . ${ }^{c}$ Used $C_{\mathrm{N}}{ }^{1}+C_{\mathrm{N}^{\prime}}{ }^{1}=1.05(0.80)$ from this work on cyclic triamines. ${ }^{d}$ Used $C_{\mathrm{N}}{ }^{1}=0.85$ and $C_{\mathrm{N}^{\prime}}{ }^{1}=0.65(0.42)$ from this work. ${ }^{e}$ Taken from ref 6.

$\mathrm{R}_{2} \mathrm{NH}_{2}{ }^{+}$groups constricted in a small cyclic molecule. The low-field chemical shifts observed for the ethylene group, $\Delta \delta=$ $0.32(\mathrm{pH} 13.5-9.5)$ and $\Delta \delta=0.34(\mathrm{pH} 9.5-5)$, give $C_{\mathrm{N}^{2}}{ }^{2}+C_{\mathrm{N}^{\prime}}{ }^{2}$ values compatible with $C_{\mathrm{N}^{2}}{ }^{2}=0.60 \mathrm{ppm}$ and $C_{\mathrm{N}^{\prime}}{ }^{2}=0.30 \mathrm{ppm}$ reported for linear amines ${ }^{8}$ (Table II). No shielding constants could be obtained for the $\mathrm{H}_{3} \mathrm{~L}^{3+}$ form because at $\mathrm{pH} 0.4$ this species is not fully formed.

The $\mathrm{pH}$ dependence of the proton chemical shifts of the ethylene protons and the methyl protons of $\mathrm{Me}_{3}[9] \mathrm{aneN}_{3}$ show three inflections at $\mathrm{pH} 11.7,5.1$, and $\simeq 0.4 \pm 0.2$ corresponding to stepwise protonation of the amino groups of macrocycle 3. Although no protonation constants for this compound have been reported, the approximate values obtained from the NMR titration curves feature a larger $\log K_{1}$ and a smaller $\log K_{2}$ than for 2 . These relative values and the absolute $\log K_{2}$ values are very different from those of [14]ane $\mathrm{N}_{4}$ and $\mathrm{Me}_{4}[14] \mathrm{aneN}_{4},{ }^{22}$ indicating that the protonation process of triaza and tetraaza cyclic amines is quite different. ${ }^{23}$ Only the sum of the methylene shielding constants $\left(C_{\mathrm{N}}{ }^{1}+C_{\mathrm{N}^{1}}{ }^{1}\right)$ could be obtained from the protonation shifts of the single ethylene peak. The observed shifts $\Delta \delta=0.35$ (pH 14-9) and $\Delta \delta=0.20(\mathrm{pH} \mathrm{9-4)}$ give values of 1.05 and $0.80 \mathrm{ppm}$ for $\left(C_{N^{1}}+C_{N^{\prime}}{ }^{1}\right)$ for the high- and low-pH regions, respectively. From the observed shifts of the methyl groups in these $\mathrm{pH}$ regions, values of $C_{\mathrm{N}}{ }^{3}=0.66 \mathrm{ppm}(\mathrm{high} \mathrm{pH})$ and $C_{\mathrm{N}}{ }^{3}=1.52($ low $\mathrm{pH})$ were also obtained.

As summarized in Table II, the $C_{i j}$ constants obtained at high and low $\mathrm{pH}$ for the nonmethylated cyclic amine 2 and linear amine systems ${ }^{8}$ agree well, indicating that the conformation of $\mathbf{2}$ is not much changed in the two first protonation steps. This is not true for the trimethylated macrocycle 3 , where the $C_{i j}$ constants are very $\mathrm{pH}$ dependent and in some cases are very different from the values obtained for linear ${ }^{8}$ and cyclic tetraaza amines. ${ }^{6}$ These discrepancies must arise from different conformations of the various protonated forms of these small methylated cyclic tri- and tetraamines. For instance, we observe that the protonation shifts of the methyl groups increase as the macrocycle becomes more protonated and also increase when the size of the macrocyclic ring

(22) Micheloni, M.: Sabatini, A.: Paoletti. P. J. J. Chem. Soc. Perkin Trans. 2 1978, 828

(23) Micheloni, M.; Paoletti, P.; Vacca, A. J. Chem. Soc., Perkin Trans. 2 1978, 945 decreases (Table II). This provides evidence for the proposal ${ }^{24}$ that the methylated cyclic amines do not form strong intramolecular hydrogen bonds when an amine is protonated and, hence, the methyl groups are preferentially directed toward the inside of the ring and consequently experience a shielding effect due to the nearby positively charged nitrogens. The unusual conformational properties of these small cycles are also expressed in the observed $\mathrm{pH}$ dependence of the line width of the ethylene resonance in the methylated cyclic amines. This has been observed before for cyclic tetraamines ${ }^{5}$ and is also seen in $\mathrm{Me}_{3}[9] \mathrm{aneN}_{3}$, where the ethylene resonance is broad for the partially protonated species, $\mathrm{HL}^{+}$and $\mathrm{H}_{2} \mathrm{~L}^{2+}$. This is most probably caused by slow interconversion between various ring conformations due to nitrogen inversion. These ring-inversion processes are less likely to contribute to the proton line width of the non-methylated cyclic amines since the conformations of the partially protonated forms are stabilized by internal hydrogen bonds. ${ }^{23}$ This problem is under further investigation.

NOTA. The $\mathrm{pH}$ dependence of the ethylene and acetate proton chemical shifts of NOTA show three inflections near pH 11.3, 5.6 , and $\sim 2.5$. The first two protonations result in shielding of the ethylene and acetate protons while the third protonation results in only a slight shielding of the acetate protons and a deshielding of the ethylene protons. We found no appreciable difference between the NMR titration curves obtained for unpurified NOTA containing two $\mathrm{NaBr}$ molecules titrated with $\mathrm{KOD}$ and for NOTA purified of any $\mathrm{Na}^{+}$by cation-exchange chromatography titrated with $\mathrm{NMe}_{4} \mathrm{OD}$, therefore indicating that, unlike in EDTA, the binding of $\mathrm{Na}^{+}$is weak enough not to affect the titration curves.

The percent protonation of the nitrogen atoms $f_{\mathrm{N}}$ and of the carboxylic oxygen atoms $f_{\mathrm{O}}$ of NOTA was calculated by using eq 1 and 2, the protonation shifts measured for $n$ number of protons added to NOTA equal to 1,2,3, and 5, and various sets of methylene group shielding constants. Table III compares the results obtained for NOTA with use of different sets of parameters from the literature for cyclic tetraamines ${ }^{6,11}$ and our own values for the cyclic triamine $\mathrm{Me}_{3}[9]$ aneN $_{3}$ (for $n<3$ the "high-pH" values of $C_{\mathrm{N}}$ and $C_{\mathrm{N}}$. were used, whereas for $n>3$ their "low-pH" values were used). It can be seen from the table that $\mathrm{Me}_{3}[9] \mathrm{aneN}_{3}$ is a very poor model for NOTA (case $c$ ), giving very large error

(24) Desreux, J. F.: Duychaerts, G. Inorg. Chim. Acta 1979, 35, 1313. 
Table IV. Average Values of $C_{\mathrm{N}}$ and $C_{\mathrm{N}^{\prime}}$ Calculated from the Titration Curves of NOTA

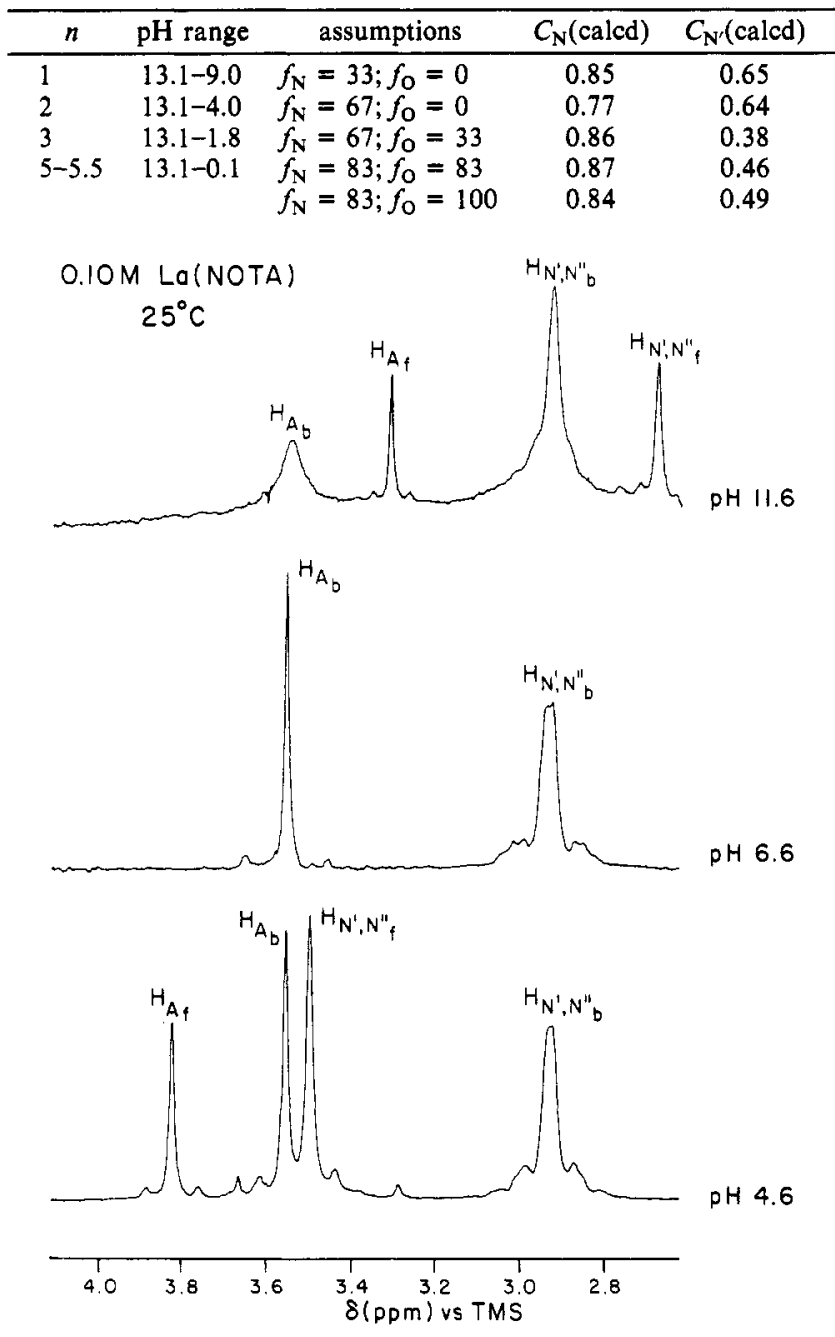

Figure 2. Proton NMR spectra of $\mathrm{La}(\mathrm{NOTA})$ at different $\mathrm{pH}$ values.

limits for $f_{\mathrm{N}}$ and $f_{\mathrm{O}}$ and unlikely results, e.g. values of $f_{\mathrm{N}}$ and $f_{\mathrm{O}}$ well over $100 \%$. The values reported for cyclic tetraamines (case $a$, ref 6 ; case $b$, ref 11 ; see Tables II and III), although better, still gave high error limits and negative $f_{\mathrm{O}}$ values. Similar discrepancies have been obtained previously for cyclic tetraaza complexes. ${ }^{\text {I }}$ In spite of this, the calculated $f_{\mathrm{N}}$ and $f_{\mathrm{O}}$ values, together with the $\mathrm{pH}$ titration curves, clearly indicate that the first 2 equiv of acid added to NOTA protonate equally all three nitrogen atoms $\left(\log K_{1}=11.3 ; \log K_{2}=5.59\right)$, and the next one protonates the carboxylate groups $\left(\log K_{3}=2.88\right)$.

We then used the observed protonation shifts of the ethylene and acetate proton resonances of NOTA to calculate the shielding constants in well-defined protonation stages, namely $n=1,2,3$, and 5 (or 5.5), assuming reasonable protonation percentages (Table IV: there is some uncertainty for the low-pH value, as even at $\mathrm{pH} 0.1$ the titration was not complete; however, several cases were considered). The calculated $C_{N}$ values changed very little with $\mathrm{pH}$, whereas $C_{\mathrm{N}^{\prime}}$ changed considerably, from a "high$\mathrm{pH}$ " value of $0.65 \mathrm{ppm}$ to "low-pH" values of $0.49-0.38 \mathrm{ppm}$. These values and changes are very different from those observed for the corresponding tetraaza macrocycles. ${ }^{6,11}$ Finally we recalculated the $f_{\mathrm{N}}$ and $f_{\mathrm{O}}$ values for NOTA using values $C_{\mathrm{N}}=0.85$ ppm (high pH and low pH) and $C_{\mathrm{N}^{\prime}}=0.65 \mathrm{ppm}$ (high pH) and $0.42 \mathrm{ppm}$ (low $\mathrm{pH}$ ) (case $d$, Table III). The protonation probabilities now have small deviations and are easily interpreted. After the two first protons protonate equally the three nitrogens, the third protonates the carboxylate oxygens between $\mathrm{pH} 4$ and 2 , causing the acetate protons to shift upfield, a clear indication ${ }^{6,10}$ that the carboxylate groups of $\mathrm{H}_{2} \mathrm{~L}^{2+}$, which are hydrogen bonded two-thirds of the time to their neighboring $\mathrm{NR}_{2} \mathrm{H}^{+}$groups, become
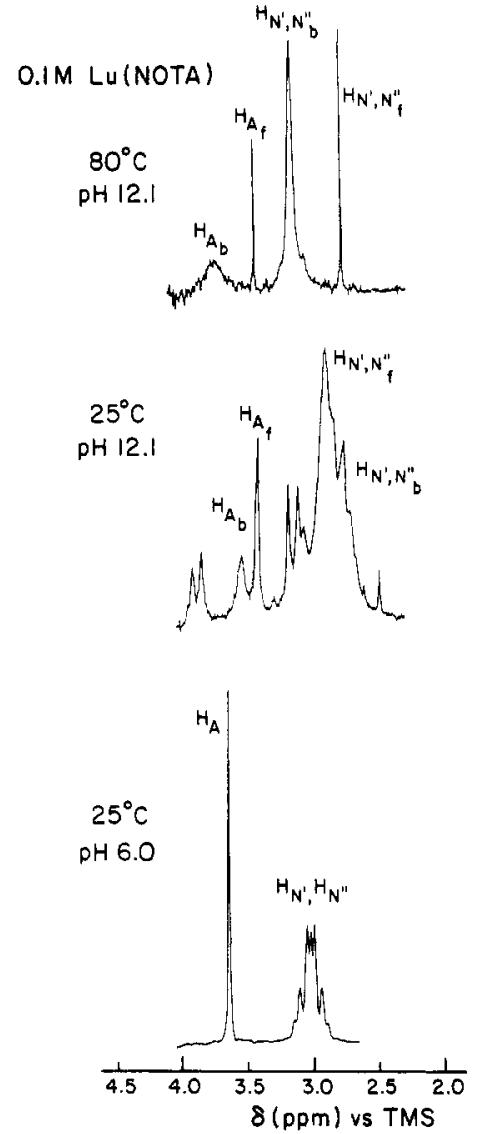

Figure 3. Proton NMR spectra of $\mathrm{Lu}(\mathrm{NOTA})$ at different $\mathrm{pH}$ values and temperatures.



Figure 4. ${ }^{13} \mathrm{C}$ NMR spectrum of $0.1 \mathrm{M} \mathrm{Lu}$ (NOTA) at $\mathrm{pH} 11.8$ and 25 ${ }^{\circ} \mathrm{C}$. The subscripts $\mathrm{a}$ and $\mathrm{h}$ refer to the aquo and hydroxo complexes, respectively.

free to rotate as they are protonated. The value of $\log K_{3}=2.88$ is markedly lower than the first two protonation constants for the acetate groups of DOTA $\left(\log K_{3}=4.24, \log K_{4}=4.18\right)$. This reflects the greater population of hydrogen-bonded acetates in the diprotonated species of NOTA vs. that of DOTA. The last three equivalents of acid bind almost simultaneously to the other carboxylate groups and to the nitrogen atoms, as shown in Table III. This protonation scheme for NOTA shares some characteristics with that obtained for DOTA ${ }^{6}$ (see Table III), including the observation that not all amino groups in these cyclic structures are more basic than the carboxylate groups. 


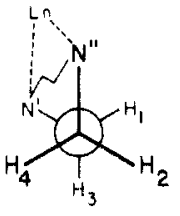

I

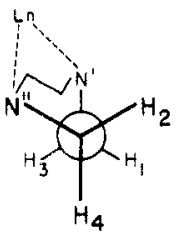

II

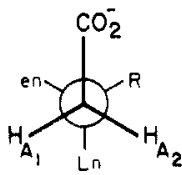

III

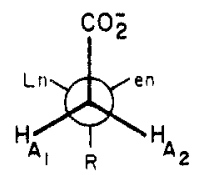

IV

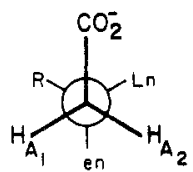

V
Figure 5. (I, II) Newman projections along the $\mathrm{C}-\mathrm{C}$ bond of the two staggered conformations of the ethylenediamine moiety of $\operatorname{Ln}(\mathrm{NOTA})$. (III-V) Newman projections along the $\mathrm{C}-\mathrm{N}$ bond of the three staggered conformations of the acetate groups of $\operatorname{Ln}(\mathrm{NOTA})$.

3. La(NOTA) and Lu(NOTA) Spectra. The proton and ${ }^{13} \mathrm{C}$ spectra of $\mathrm{La}$ (NOTA) and Lu(NOTA), recorded at various temperatures and $\mathrm{pH}$ values, are reproduced in Figures 2-4. The proton spectrum of the La(NOTA) (1:1) complex shows two signals, corresponding to the ethylenic and acetate protons, only over a limited $\mathrm{pH}$ range. Below $\mathrm{pH} 6$ and above $\mathrm{pH} 10.6$, two new sharp singlets, corresponding to free NOTA resonances, can be seen (Figure 2). Between $\mathrm{pH} 6$ and pH 9.5, the La(NOTA) acetate proton signal is a sharp singlet and the ethylenic protons give a symmetrical $\mathbf{A A}^{\prime} \mathbf{B B}^{\prime}$ splitting pattern. However, above $\mathrm{pH} 9.5$ these two signals start to broaden, as a consequence of formation of a La(NOTA) hydroxo complex. The Lu(NOTA) complex is stable over a much wider $\mathrm{pH}$ range than the $\mathrm{La}^{3+}$ analogue, as indicated by its proton and ${ }^{13} \mathrm{C}$ NMR spectra (Figures 3 and 4 ), which show no evidence of free NOTA in solution between pH 2 and 11.8. From pH 2 to 9.2 there is only one Lu(NOTA) complex in solution, giving a proton spectrum consisting of a sharp singlet for the acetate protons and a symmetrical $\mathrm{AA}^{\prime} \mathrm{BB}^{\prime}$ pattern for the ethylenic protons, whereas the ${ }^{13} \mathrm{C}$ spectrum consists of three sharp singlets representing the carboxylate, acetate, and ethylenic carbons. However, at pHs above 9.2 , a Lu(NOTA) hydroxo complex begins to form and is the dominant form by $\mathrm{pH} 11.8$. Its proton and ${ }^{13} \mathrm{C}$ spectra are much more complex than the spectrum of the Lu(NOTA) aquo complex, giving a proton spectrum consisting of an $\mathrm{AB}$ quartet for the acetate protons $(J=15.8 \mathrm{~Hz})$ and a complex $\mathrm{ABCD}$ pattern for the ethylenediamine protons (Figure 3 ) and a ${ }^{13} \mathrm{C}$ spectrum consisting of singlets for the carboxyl and acetate carbons and a doublet for the ethylenic carbons (Figure 4). A temperature increase collapses the multiplet patterns of the acetate and ethylenic proton spectra to a singlet and an $\mathbf{A A}^{\prime} \mathbf{B B}^{\prime}$ pattern, respectively, and the ethylenic carbon doublet to a singlet (data not shown). The ${ }^{13} \mathrm{C}$ spectra of the Lu(NOTA) aquo and hydroxo species are in slow exchange at all temperatures.

The ${ }^{1} \mathrm{H}$ and ${ }^{13} \mathrm{C}$ spectra of aqueous $\mathrm{La}(\mathrm{NOTA})$ and $\mathrm{Lu}-$ (NOTA) indicate that the triaza macrocyclic ring structures are quite flexible in these chelates. As the nine-membered macrocycle ring is too small to accommodate a lanthanide ion in its internal cavity, the metal ion can be assumed to be placed above the plane formed by the three nitrogen atoms. ${ }^{12}$ Although all carbon and hydrogen nuclei are nonequivalent in conformations I or II of the ethylenediamine moiety (Figure 5), rapid equilibration between these two staggered conformations interconverts protons $\mathrm{H}_{1}$ and $\mathrm{H}_{2}$ and protons $\mathrm{H}_{3}$ and $\mathrm{H}_{4}$. Therefore, the observation of an ethylenic proton $\mathrm{AA}^{\prime} \mathrm{BB}^{\prime}$ splitting pattern for the aqueous $\mathrm{La}$ (NOTA) and Lu(NOTA) complexes shows that the interconversion between the two staggered conformations I and II is rapid. This conclusion is supported by the ${ }^{13} \mathrm{C}$ NMR data. Although flexible ethylenediamine moieties have been observed for the sandwich-type lanthanide complexes of the tetraoxa ligand 12 crown- $4 .{ }^{24}$ they have been shown to be rigid at room temperature in the tetraaza macrocyclic $\mathrm{La}(\mathrm{DOTA})$ and $\mathrm{Lu}(\mathrm{DOTA})$ complexes. $^{13}$

Formation of the hydroxo form of the Lu(NOTA) complex dramatically increases the rigidity of the bound NOTA, leading, even at room temperature, to a doublet in the ${ }^{13} \mathrm{C}$ spectrum (Figure 4) and a complex $\mathrm{ABCD}$ pattern in the ${ }^{1} \mathrm{H}$ spectrum (Figure 3) of the ethylenic groups. Poor resolution at this high $\mathrm{pH}$ precluded a quantitative analysis of this complex splitting pattern. The La(NOTA) hydroxo complex shows only broad resonances, indicating that the exchange between ethylenediamine conformers is considerably more rapid in this complex.

Although the barrier to interconversion of ethylenediamine conformers could in principle be obtained by a band-shape analysis of the ${ }^{13} \mathrm{C}$ resonances in the Lu(NOTA) hydroxo complex, this was not undertaken since this doublet is always superimposed on the intense singlet resulting from the aqueous complex (Figure 4). However, an estimate of $\Delta G, \simeq 64 \pm 2 \mathrm{~kJ} \mathrm{~mol}^{-1}$, could be obtained from the coalescence temperature, $T_{\mathrm{c}} \simeq 45^{\circ} \mathrm{C}$.

The appearance of the acetate proton resonances in diamagnetic complexes of polyamino polycarboxylates has been interpreted in terms of the relative lifetimes of the metal-amine and metal-oxygen bonds..$^{25-30}$ If both lifetimes are short on the NMR time scale, the acetate spectrum consists of a singlet. If, however, the metal-nitrogen bond lifetime is long while the metal-oxygen bond is labile, inversion through the nitrogen atom is not possible and the nonequivalent acetate protons $\mathrm{H}_{\mathrm{A}_{1}}$ and $\mathrm{H}_{\mathrm{A}_{2}}$ (see structures III, IV, and $\mathrm{V}$ in Figure 5) give $\mathrm{AB}$ quartets. Indeed, a quartet pattern is observed for the acetate protons in Lu(EDTA) ${ }^{28,29}$ ( R $\left.=\mathrm{CH}_{2} \mathrm{CO}_{2}^{-}\right)$and in La(DOTA) and Lu(DOTA) at low temperature $^{13}(R=$ en $)$. In the latter case, the low-temperature quarternary nitrogen asymmetry was shown to derive from the rigidity and conformational preferences of the tetraaza macrocycle. When the temperature was increased, the ethylenediamine groups interconverted rapidly on the NMR time scale, leading to a systematic quarternary nitrogen and the equivalent acetate protons gave a singlet resonance. In the present study, the equivalence of the acetate protons in the aqueous La(NOTA) and Lu(NOTA) chelates at room temperature indicates that either the metalnitrogen bonds are labile in these complexes, perhaps resulting from the very small internal cavity of the triaza macrocyclic ring, or rapid equilibration of the ethylenediamine ring conformations result in an averaging of the magnetically nonequivalent acetate protons. The hydroxo complex of Lu(NOTA) displays an AB quartet at room temperature, which collapses into a singlet at the same higher temperature that collapses the ethylenic carbon doublet into a singlet. These observations again illustrate the rigidity of this complex and support the view that the nonequivalence of the acetate protons is due to the stereochemical behavior of the triaza macrocyclic ring. Again the La(NOTA) hydroxo complex is less rigid than the Lu complex, as at room temperature the acetate singlet, although broadened, does not split into an $\mathrm{AB}$ quartet.

\section{Conclusions}

The present NMR and potentiometric investigation of NOTA shows that this triaza macrocyclic complexon contains one nitrogen atom which is less basic than at least one of its carboxylate groups. This property is shared by cyclic tetraaza polycarboxylates, ${ }^{6,7}$ but not by linear complexons like EDTA . $^{8,10}$ The protonation sequence of NOTA has been determined with use of shielding constants obtained for well-defined protonation stages, as the values for these parameters deduced from studies of linear systems, ${ }^{8}$ the cyclic triamines, or cyclic tetraaza complexons ${ }^{6,7}$ give inconsistent and unrealistic percent protonation values. In fact, the shielding

(25) Kula, R. J.; Sawyer, D. T.; Chan, S. I.; Finley, C. M. J. Am. Chem. Soc. 1963, 85, 2930.

(26) Day, R. J.; Reilley, C. N. Anal. Chem. 1964, 36, 1073.

(27) Day, R. J.; Reilley, C. N. Anal. Chem. 1965, 37, 1326

(28) Kostromina, N. A.; Tevnovaya, T. V. Teor. Eksp. Khim. 1971, 7, 115.

(29) Ryhl, T. Acta Chem. Scand. 1972, 26, 4001.

(30) Baisden, P. A.; Choppin, G. R.; Garrett, B. B. Inorg. Chem. 1977, 16, 1367. 
constants $C_{\mathrm{N}}$ and $C_{\mathrm{N}^{\prime}}$ of the ethylenic protons and of the acetate protons in these cyclic structures change with $\mathrm{pH}$ in a different way depending on the type of proton considered. ${ }^{6.7}$ This results from changes in conformational equilibria of the various groups caused by restricted flexibility, electrostatic repulsions and hydrogen bond formation that are difficult to predict a priori. These restrictions seem to be even more severe in the cyclic triaza macrocycles than in the tetraaza analogues.

The constants $\log K_{1}$ and $\log K_{2}$ refer to equal statistical protonation of the nitrogen atoms in the ring. The constant log $K_{3}=2.88$ is similar to $\log K_{3}$ for EDTA and remarkably lower than the values $\log K_{3}$ and $\log K_{4}$ reported for DOTA, which reflect the protonation of the two carboxylate moieties not adjacent to a protonated nitrogen atoms in the $\mathrm{H}_{2} \mathrm{~L}^{2+}$ form of DOTA. The $\log K_{3}$ value in NOTA, which refers to protonation of the first carboxylate group in the $\mathrm{H}_{2} \mathrm{~L}^{2+}$ form, is lower because each carboxylate group is $66 \%$ hydrogen bonded to an adjacent nitrogen atom which bears $66 \%$ of a positive charge. The last three protonation constants, which reflect protonation of the remaining two carboxylates and a single ring nitrogen, are even lower for two reasons. The two carboxylate groups are now fully hydrogen bonded to their adjacent positively charged nitrogens while protonation of the last nitrogen is constrained by the electrostatic repulsions imposed by a nine-membered ring containing three positively charged nitrogens.

There are conformational similarities between the lanthanide complexes of the polyaza macrocycles, NOTA and DOTA, and the hydrocarbon analogues of these cycles. It has been shown ${ }^{13}$ that the tetraaza cycle of DOTA, like the 12 -membered ring of cyclododecane, ${ }^{31,34}$ has two favored enantiomeric square [3333]

(31) Dale, J. Acta Chem. Scand. 1973, 1115, 1130

(32) Anet, F. A. L.; Wagner, J. J. J. Am. Chem. Soc. 1971, 93, 5266.

(33) Anet, F. A. L.; Cheng, A. K.; Wagner, J. J. J. Am. Chem. Soc. 1972. 94,9250 . conformations, whereas both the triaza cycle of NOTA and the 9-membered ring of cyclononane have two favored enantiomeric triangular [333] conformations (each enantiomer has four or three identical ethylenic groups with conformations $\delta$ or $\lambda$ ). Previous dynamic NMR measurements ${ }^{32,39}$ have shown that the energy barrier for interconversion between these ring conformations is smaller in cyclononane $\left(25 \mathrm{~kJ} \mathrm{~mol}^{-1}\right)$ than in cyclododecane $(30.5$ $\mathrm{kJ} \mathrm{mol}^{-1}$ ). This same trend is preserved in the lanthanide complexes of NOTA vs. those of DOTA. The NMR data reported in this work for $\mathrm{La}$ (NOTA) and $\mathrm{Lu}$ (NOTA) shows that the 9 -membered ring in these complexes is much more flexible than the 12-membered ring in the corresponding DOTA complexes. ${ }^{13}$ Although the barrier for ethylene ring conformational changes is similar in $\mathrm{La}$ (DOTA) $\left(60.7 \mathrm{~kJ} \mathrm{~mol}^{-1}\right)^{13}$ and the hydroxo complex of Lu(NOTA) $\left(\sim 64 \mathrm{~kJ} \mathrm{~mol}^{-1}\right)$, the smaller triaza macrocycle requires the smaller lanthanide cation, $\mathrm{Lu}^{3+}$, for structural rigidity. Also a correlation is found between the conformational rigidity of these complexes and their kinetics of formation; i.e., the NOTA complexes are flexible and somewhat more labile while the DOTA complexes are rigid and quite inert. Therefore, the conformational characteristics of the cyclic ring in these chelated ligands are, in part, determined by the extent of encapsulation of a lanthanide cation in addition to its own steric requirements.

Acknowledgment. The authors thank Professor Greg Choppin and Bill Cacheris of Florida State University for performing the potentiometric titrations. This investigation was supported by Grant AT-584 from the Robert A. Welch Foundation and by NATO Travel Grant No. 0345/82. C.F.G.C.G. acknowledges support from INIC, Portugal, and M.S. thanks Guru Nanak Dev University for a study leave.

Registry No. 1, 56491-86-2; 3, 96556-05-7; La(NOTA), 78085-47-9; Lu(NOTA), 98331-80-7.

(34) Anet, F. A. L.; Rawdah, T. W. J. Am. Chem. Soc. 1978, 100, 7166.

Contribution from the Department of Materials Science and Engineering, Massachusetts Institute of Technology, Cambridge, Massachusetts 02139

\title{
Raman Spectroscopic Investigation of Alkali-Metal Hexachloro Compounds of Refractory Metals
}

\author{
G. J. KIPOUROS, ${ }^{1 a}$ J. H. FLINT, and D. R. SADOWAY*1b
}

Received January 15, 1985

The Raman spectra of molten alkali-metal hexachlorozirconate, hexachlorohafnate, hexachloroniobate, and hexachlorotantalate compounds have been obtained in the temperature range 623-1143 K. The results confirm that the refractory metal exists in the form of an octahedrally coordinated complex anion that is stable even in the molten state. For a given refractory metal the frequency of the $\nu_{1}$ line increases as the size of the alkali-metal cation decreases. For a given alkali metal the frequency of the $\nu_{1}$ line increases as the valence of the refractory metal increases. This last observation may serve as the basis for detecting, by Raman spectrocopy, aliovalent species that may form during the electrolysis of melts containing refractory-metal chlorides.

\section{Introduction}

The chlorides of the elements of groups 4 and $5^{22}$ react with alkali-metal chlorides to produce hexachloro compounds of the general formulas $\mathrm{A}_{2} \mathrm{MCl}_{6}$ and $\mathrm{ANCl}_{6}$, where $\mathrm{A}$ is an alkali metal, $\mathrm{M}$ is a group 4 metal, and $\mathrm{N}$ is a group 5 metal. The volatile covalently bonded refractory-metal chlorides exist in thermodynamically stable forms in these compounds, which, when dissolved in alkali-metal chloride melts, constitute potential electrolytes for the electrodeposition of the refractory metals. However, their electrolytic recovery is impaired by the formation of aliovalent species, which are difficult to identify during electrolysis.

(1) (a) Present address: General Motors Research Laboratories, Electrochemistry Department, Warren, MI 48090. (b) To whom correspondence should be addressed.
In order to determine whether Raman spectroscopy can be useful in this regard, the Raman spectra of melts of the following compounds were measured: $\mathrm{Na}_{2} \mathrm{ZrCl}_{6}, \mathrm{~K}_{2} \mathrm{ZrCl}_{6}, \mathrm{Cs}_{2} \mathrm{ZrCl}_{6}$, $\mathrm{Li}_{2} \mathrm{HfCl}_{6}, \mathrm{Na}_{2} \mathrm{HfCl}_{6}, \mathrm{~K}_{2} \mathrm{HfCl}_{6}, \mathrm{Cs}_{2} \mathrm{HfCl}_{6}, \mathrm{KNbCl}_{6}, \mathrm{CsNbCl}$, $\mathrm{NaTaCl}_{6}, \mathrm{KTaCl}_{6}, \mathrm{CsTaCl}_{6}$.

\section{Experimental Section}

The zirconium and hafnium hexachloro compounds were synthesized in this laboratory by the reaction of $\mathrm{ZrCl}_{4}$ or $\mathrm{HfCl}_{4}$ vapor with solid alkali-metal chloride under controlled pressure and temperature conditions. The preparation has been described elsewhere..$^{2-5}$ The niobium

(2) Lister, R. L.; Flengas, S. N. Can. J. Chem. 1963, 4l, 1548.

(3) Kipouros, G. J.; Flengas, S. N. Can J. Chem. 1978, 56, 1549.

(4) Kipouros, G. J.; Flengas, S. N. Can. J. Chem. 1981, 59, 990

(5) Kipouros, G. J.; Flengas, S. N. Can J. Chem. 1983, 61, 2183. 